\title{
Perioperative internal thoracic artery steal syndrome after coronary bypass surgery
}

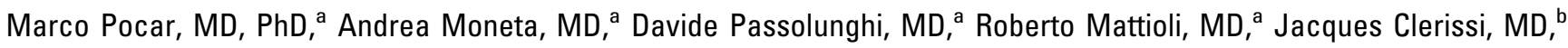 \\ and Francesco Donatelli, MD, ${ }^{a}$ Milan, Italy
}

T he internal thoracic artery (ITA) flow steal syndrome after coronary bypass operations is rare. In the absence of subclavian artery stenosis, it has mainly been attributed to incomplete harvesting of the conduit along its proximal course. ${ }^{1}$ Previous reports describe the recurrence of angina months or years after revascularization. The possibility of an ITA steal phenomenon associated with acute transmural myocardial ischemia occurring perioperatively is presented.

\section{Clinical Summary}

A 73-year-old, hypertensive, non-insulin-dependent diabetic man with normal left ventricular function and no history of prior myocardial infarction or evidence of pulmonary disease underwent triple bypass grafting for worsening anginal symptoms caused by three-vessel coronary disease. The operation was performed with conventional cardioplegic arrest, and included left ITA grafting to the left anterior descending artery and saphenous vein bypass to the obtuse marginal and right posterior descending branches. The patient was weaned from cardiopulmonary bypass with no inotropic support or sign of myocardial ischemia. Extracorporeal circulation and aortic crossclamp times were 96 and 57 minutes, respectively.

Three hours after arrival in the intensive care unit, the patient showed nonsustained ventricular tachycardia, immediately followed by signs of acute transmural anterolateral ischemia. The electrocardiogram gradually normalized with nitroglycerin infusion; intravenous amiodarone was also administered. No further instability occurred, and extubation was accomplished after control coronary angiography, which showed patent bypass grafts and an undivided collateral branch originating from the proximal ITA (Figure 1, $A$ and $B$ ). Thereafter, however, blood gas exchanges remained borderline, and despite the application of continuous positive airway pressure, the patient subsequently experienced a low output state (oliguria, serum liver-specific enzyme increase, and paroxysmal supraventricular tachyarrhythmias) and eventually required reintubation for mechanical ventilatory support. The peak

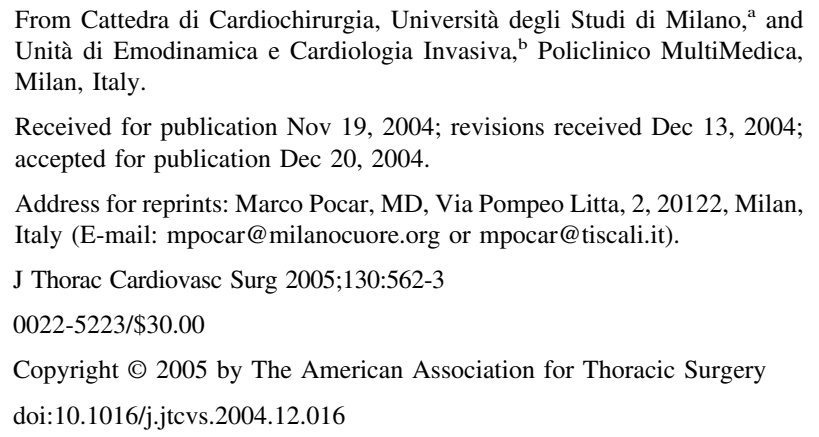

serum creatine kinase (myocardial band) level was 1139 (90) U/L. Catheterization was repeated on the basis of the previous angiograms to perform coil embolization of the ITA collateral branch (Figure 1, C).

Cardiorespiratory function improved, and the patient was extubated the following day, showing no further complications. Echocardiography documented hypokinesia of the left ventricular apex with normal ejection fraction, and the patient was discharged on postoperative day 12. At 3 months' follow-up, dobutamine stress echocardiography showed no inducible wall-motion abnormality and increased diastolic ITA flow, indicating a normal-functioning graft. ${ }^{2}$

\section{Discussion}

Although the interruption of patent collaterals (with percutaneous embolization or thoracoscopic or open surgical intervention ${ }^{3,4}$ ) along the proximal course of the ITA has been described to treat recurrent chronic angina after coronary bypass operations, an acute coronary syndrome related to an ITA steal occurring perioperatively has not been reported.

The ITA steal is probably multifactorial but has essentially been attributed to partial harvesting of the conduit, which leaves one or more undivided collaterals. ${ }^{1}$ However, the possibility of a steal phenomenon on this pathophysiologic basis has been questioned. A collateral circulation alone is insufficient to explain the ITA steal syndrome satisfactorily because of the nature of coronary blood flow to the left ventricle, which, unlike blood flow to the chest wall and upper extremities, is predominantly diastolic. ${ }^{5}$

Previous reports usually have described large collaterals near the ITA origin, which might show unusual takeoff angles from the ITA and sometimes represent true anatomic variants. Increased resistance to blood flow might also favor a steal phenomenon in spite of a patent anastomosis and a rapid washout of contrast medium at coronary angiography. This can occur in the presence of peripheral coronary disease, kinking or spasm of the ITA graft, or left ventricular overdistension. Our patient actually had transmural ischemia immediately after an episode of ventricular tachycardia, which correlates well with cardiac distension, and was diabetic, indicating a higher possibility of peripheral vascular disease. A coronary or ITA spasm might also have occurred transiently. In summary, it is virtually impossible to determine the event that started the vicious circle, but most probably the triggering cause was either purely ischemic and thus related to borderline ITA flow because of the patent collateral, or possibly primarily electric.

Finally, the ITA steal phenomenon has recently gained interest in relation to less-invasive coronary operations performed through a minithoracotomy or ministernotomy because only incomplete harvesting of the conduit is provided unless thoracoscopy is used to dissect the proximal ITA. Interestingly, myocardial ischemia generally does not develop in these patients, indicating that the 

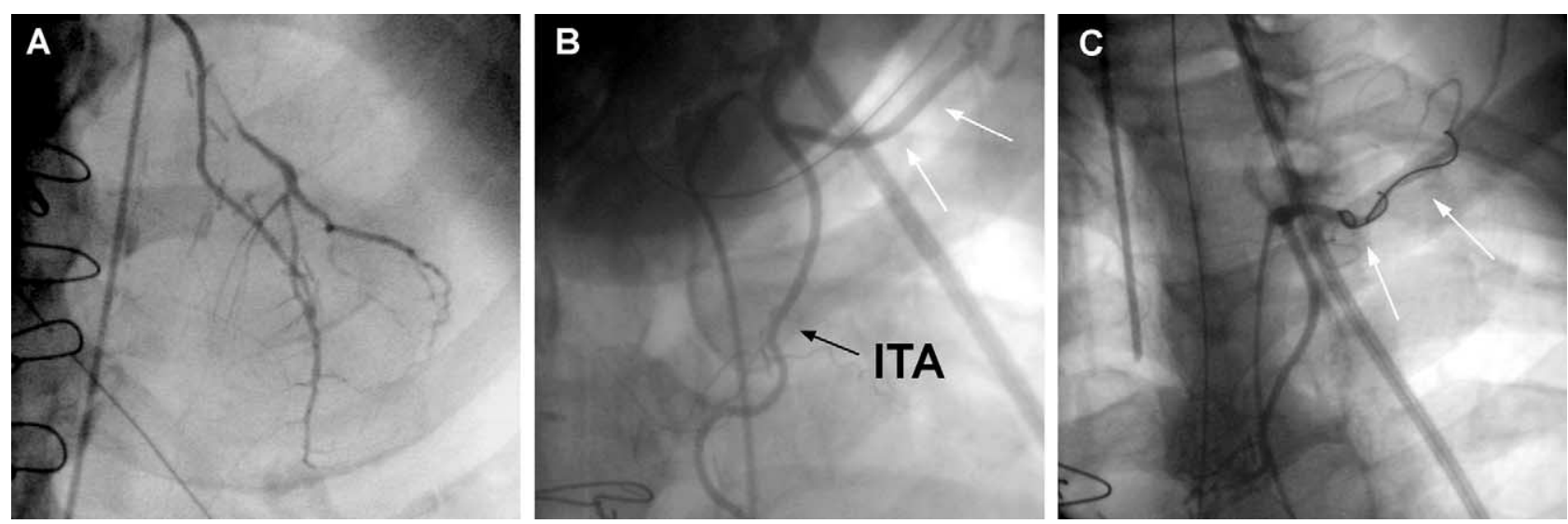

Figure 1. Angiograms showing a patent bypass graft $(A)$ and an undivided collateral branch arising from the proximal course of the left ITA before (B) and after (C) coil embolization.

presence of an unusually large collateral is probably necessary for the steal to occur.

\section{Conclusion}

Acute myocardial ischemia might be related to an ITA steal syndrome. The condition is probably uncommon, but in view of its implications and the prevalence of coronary operations, an undivided or aberrant ITA collateral should be suspected in case of unexplained perioperative myocardial ischemia.

\section{References}

1. Tonz M, von Segesser L, Carrel T, Pasic M, Turina M. Steal syndrome after internal mammary artery bypass grafting - an entity with increasing significance. Thorac Cardiovasc Surg. 1993;41:112-7.
2. Arruda AM, Pellikka PA, Mahoney DW, Joseph A Jr, Mathias W Jr, Seward JB. Transthoracic Doppler echocardiographic comparison of left internal mammary artery grafts to left anterior descending coronary artery with ungrafted right internal mammary arteries in patients with and without myocardial ischemia by dobutamine stress echocardiography. Am J Cardiol. 2000;86:919-22.

3. Nakhjavan FK, Koolpe HA, Bruss J, Najmi M, Radke T. Transcatheter coil occlusion for the treatment of left internal mammary-anterior descending artery steal phenomenon. Catheter Cardiovasc Diagn. 1993; 28:347-50.

4. Pagni S, Bousamra M 2nd, Shirley MW, Spence PA. Successful VATS ligation of a large anomalous branch producing IMA steal syndrome after MIDCAB. Ann Thorac Surg. 2001;71:1681-2.

5. Gaudino M, Serricchio M, Tondi P, Glieca F, Bruno P, Possati G, et al. Do internal mammary artery side-branches have the potential for haemodynamically significant flow steal? Eur J Cardiothorac Surg. 1999; 15:251-5. 